\title{
Estrogen-inducible GFP expression patterns in rice (Oryza sativa $\mathbf{L}$.)
}

\author{
Ayako Okuzaki • Ken-ichi Konagaya • \\ Yoshihiko Nanasato $\cdot$ Mai Tsuda $\cdot$ Yutaka Tabei
}

Received: 13 September 2010/Revised: 5 November 2010/Accepted: 23 November 2010/Published online: 8 December 2010

(C) The Author(s) 2010. This article is published with open access at Springerlink.com

\begin{abstract}
We investigated estrogen-inducible green fluorescent protein (GFP) expression patterns using an estrogen receptor fused chimeric transcription activator, $\mathrm{XVE}$, in the monocotyledonous model plant rice (Oryza sativa $\mathrm{L}$.). This system has been shown to be an effective chemical-inducible gene expression system in Arabidopsis and has been applied to other plants in order to investigate gene functions or produce marker-free transgenic plants. However, limited information is available on the correlation between inducer concentration and the expression level of the gene induced in monocots. Here, we produced a transgenic rice integrated estrogen-inducible GFP expression vector, $p L e x: G F P$, and investigated doseresponse and time-course patterns of GFP induction in rice calli and seedlings for the first time. With $17-\beta$-estradiol treatment at $>5 \mu \mathrm{M}$, GFP signals were detected in the entire surface of calli within 2 days of culture. Highest GFP signals were extended for 8 days with estradiol treatment at $25 \mu \mathrm{M}$. In three-leaf-stage seedlings, GFP signals in the leaves of $p L e x: G F P$-integrated transgenic lines were weaker than those in the leaves of $p 35 S: G F P$ integrated transgenic lines. However, GFP signals in the roots of pLex:GFP- and $p 35 S: G F P$-integrated transgenic
\end{abstract}

Communicated by K. Toriyama.

A. Okuzaki $\cdot$ K. Konagaya .

Y. Nanasato · M. Tsuda · Y. Tabei ( $\square)$

Division of Plant Sciences, National Institute of Agrobiological Sciences, 2-1-2 Kannondai, Tsukuba, Ibaraki 305-8602, Japan e-mail: tabei@affrc.go.jp

Present Address:

K. Konagaya

Forest Bio-Research Laboratory 1, Forest Bio-Research Center,

Forestry and Forest Products Research Institute,

3809-1 Ishi, Juo, Hitachi, Ibaraki 319-1301, Japan lines were similar with estradiol treatment at $>10 \mu \mathrm{M}$. With regard to controlling appropriate gene expression, these results might provide helpful indications on estradiol treatment conditions to be used for the XVE system in rice and other monocots.

Keywords Estrogen inducible XVE system . Estradiol · Rice · GFP

$\begin{array}{ll}\text { Abbreviations } \\ \text { GFP } & \text { Green fluorescent protein } \\ \text { Estradiol } & 17-\beta \text {-Estradiol } \\ \text { hpt } & \text { Hygromycin phosphotransferase } \\ \text { PCR } & \begin{array}{l}\text { Polymerase chain reaction } \\ \text { XVE }\end{array} \\ & \begin{array}{l}\text { An estrogen receptor fused chimeric } \\ \text { transcription activator }\end{array}\end{array}$

\section{Introduction}

Chemical-inducible gene expression systems are powerful tools for controlling the timing and level of target gene expression in plants. Chemical-inducible systems have been applied to genetic engineering techniques such as the production of marker-free transgenic plants (Zuo et al. 2001; Sreekala et al. 2005; Zhang et al. 2006; Costa et al. 2009) and the inductive production of extra protein (Dohi et al. 2006). Several chemical-inducible systems have been developed using various chemical inducers, such as tetracycline, glucocorticoid, and ethanol (Zuo and Chua 2000; Padidam 2003; Tang et al. 2004). A valuable chemicalinducible system is the one developed by Aoyama and Chua (1997) using a glucocorticoid-inducible chimeric 
transcription activator called GVG. However, growth defects in Arabidopsis (Kang et al. 1999) and rice (Ouwerkerk et al. 2001) have been attributed to GVGinduced ectopic expression of the endogenous gene. Therefore, the XVE system (Zuo et al. 2000a) has been developed as an alternative to the GVG system. Zuo et al. (2000a) demonstrated that $17-\beta$-estradiol, which is one of the estrogens, induces efficient target gene expression without causing growth defects in Arabidopsis. XVE, which is used as an estrogen responsive DNA activator, consists of the bacterial repressor LexA, herpes simplex virus protein 16 , and human estrogen receptor. The XVE system has been applied to other plants, such as tobacco BY2 cells (Zuo et al. 2000b), tomato (Zhang et al. 2006), and catharanthus (Xu and Dong 2007). In addition, the chemical-inducible XVE system is a useful tool for determining the function of genes that cannot be examined by their overexpression in plants, such as lethal genes (Xu and Dong 2007) or genes affecting morphology or causing growth defects (Petrasek et al. 2006; Klimaszewska et al. 2010). Petrasek et al. (2006) investigated the function of plant-specific pin-formed proteins. Using the XVE system, Xu and Dong (2007) demonstrated that the mammalian Bax, which triggers a hypersensitive reaction when expressed in plants, enhances terpenoid indole alkaloid production in Catharanthus roseus cells. Klimaszewska et al. (2010) examined how Arabidopsis WUS and conifer LEC1 (CHAP3A) act in transgenic white spruce using a modified XVE system.

Two reports showed that the XVE system is also functional in rice (Sreekala et al. 2005; Xu et al. 2009). However, limited information is available on the correlation between the efficacy of estradiol treatment and levels of target gene expression induced by the XVE system in rice.

Chemical-inducible gene expression systems have been applied to various rice research fields, and seem ideal to be used in rice calli for investigating complicated gene functions or produce marker-free plants. To appropriately control the expression levels of various target genes, we investigated the correlation between the efficacy of estradiol treatment and detailed analysis of gene expression levels induced by the XVE system in rice.

\section{Materials and methods}

\section{Construction of $p L e x: G F P$}

pLex:GFP (Fig. 1) was constructed as follows. The pLex:XVE:T3A cassette region was amplified by PCR from pER8 (Zuo et al. 2000a) using GXL polymerase (Takara Bio Inc., Shiga, Japan) with a set of primers (5'-GGTA CCATAGTTTAAACTGAAGGCGG-3'/5'-GTCGACGTT TGGGATGTTTTACTCCTC- $3^{\prime}$ ). The amplified PCR
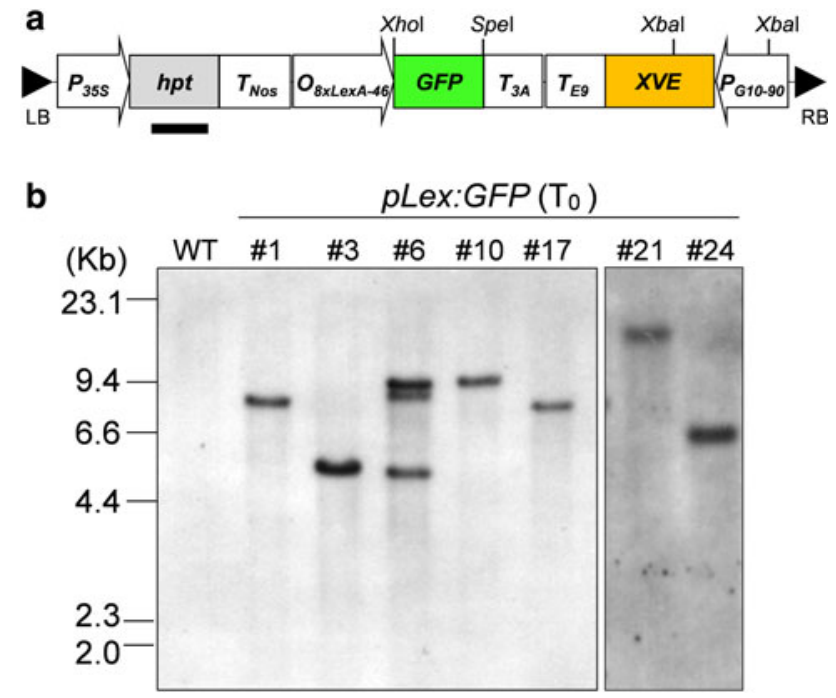

Fig. 1 Production of the pLex:GFP-integrated transgenic plants. a The pLex:GFP cassette between the right and left borders (not to scale) (a). $P_{35 S}$ CaMV $35 \mathrm{~S}$ promoter, $h p t$ hygromycin phosphotransferase, $T_{\text {Nos }} 3^{\prime}$ signal of the nopaline synthase terminator, $O_{8 x l e x A-46} 8$ copies of the lexA DNA-binding site fused to the -46 CaMV $35 \mathrm{~S}$ minipromoter, GFP sGFP (Niwa et al. 1999), $T_{3 A}$ the rbcsS $3 A$ poly(A) addition sequence, $T_{E 9}$ the $r b c S$ E9 poly(A) addition sequence, $X V E$ chimeric transactivator containing the regulator domain of an estrogen receptor (Zuo et al. 2000b), $P_{G 10-90}$ a synthetic promoter (Ishige et al. 1999), $R B$ right border and $L B$ left border. The black bar indicates the DIG-labeled hpt probe used for Southern blot analysis. b Southern blot analysis of the $p L e x: G F P$-integrated plants. DNA was digested with $X b a I$ and hybridized with the DIG-labeled hpt probe

fragment was then inserted into $p C R$-Blunt II-TOPO (Invitrogen, Carlsbad, CA, USA) to yield the plasmid $p X V E$. The $p L e x: X V E: T 3 A$ cassette was digested from $p X V E$ using KpnI and SalI and cloned into KpnI- and SalIdigested $p Z H 2 B$ (Kuroda et al. 2010), which contained $p P Z P$ (Hajdukiewicz et al. 1994) as the vector backbone, to yield the plasmid $p Z H-X V E$. The $S p h \mathrm{I}-K p n \mathrm{I}$ fragment from pER8 was cloned into $p U C 198 A M$ (Kuroda et al. 2010). Full-length GFP (Niwa et al. 1999) was amplified by PCR using GXL polymerase with a set of primers (5'-CTCGAG ATGGTGAGCAAGGGCGAG-3 $3^{\prime} / 5^{\prime}$-ACTAGTTTACTTG TACAGCTCGTCCATGCC-3') and inserted into $p C R$ Blunt II-TOPO to yield the plasmid pXhoI-GFP-SpeI. The XhoI-GFP-SpeI fragment was digested from $\mathrm{pXhoI-GFP-}$ SpeI and cloned into XhoI- and SpeI-digested pPlex-Ter to yield the plasmid pLex:GFP-T. The AscI-PacI fragment from $p L e x: G F P-T$ was cloned into AscI- and $P a c \mathrm{I}$-digested $p Z H-X V E$ to yield the final binary vector $p L e x: G F P$, which was transferred into Agrobacterium tumefaciens strain EHA105 (Hood et al. 1993). To obtain a positive control constitutively expressing GFP, the 35SP:GFP:NosT cassette was inserted into the HindIII/EcoRI site of $p Z H 2 B$ to yield $p 35 S: G F P$. 
Plant materials and production of transgenic rice harboring pLex:GFP

The binary vectors $p L e x: G F P$ (Fig. 1a) and p35S:GFP were introduced into rice (Oryza sativa L. cv. Nipponbare) using the Agrobacterium transformation system (Toki et al. 2006). To select transgenic plants, calli were cultured on N6D (Chu et al. 1975) medium containing $30 \mathrm{mg} \mathrm{L}^{-1}$ hygromycin (Wako, Osaka, Japan) and $20 \mathrm{mg} \mathrm{L}^{-1}$ meropenem (Dainippon Sumitomo Pharma, Osaka, Japan); the medium was solidified with $0.3 \%$ Gelrite. Then hygromycin-resistant calli were transferred to regeneration medium containing $30 \mathrm{mg} \mathrm{L}^{-1}$ hygromycin and $20 \mathrm{mg} \mathrm{L}^{-1}$ meropenem. Regenerated shoots were rooted on hormone-free MS medium containing $30 \mathrm{mg} \mathrm{L}^{-1}$ hygromycin; the medium was solidified with $0.8 \%$ agar. Regenerated plants were grown in a greenhouse.

Gene induction by estradiol treatment

Rice seeds were sterilized with $70 \%$ ethanol for $1 \mathrm{~min}$ and $2.5 \%$ sodium hypochlorite for $30 \mathrm{~min}$. To induce calli, the sterilized seeds were cultured on N6D medium containing $30 \mathrm{mg} \mathrm{L}^{-1}$ hygromycin under continuous light $\left(200 \mu \mathrm{mol} \mathrm{m} \mathrm{m}^{-2} \mathrm{~s}^{-1}\right)$ at $30^{\circ} \mathrm{C}$; the medium was solidified with $0.3 \%$ gelrite and was changed every 2 weeks. To prepare seedlings, the sterilized seeds were cultured on MS medium containing $30 \mathrm{mg} \mathrm{L}^{-1}$ hygromycin under continuous light at $30^{\circ} \mathrm{C}$ for 10 days; the medium was solidified with $0.8 \%$ agar. Hygromycin-resistant seedlings were transferred to Agripot (Iwaki glass, Tokyo, Japan) and were cultivated in MS liquid culture medium with or without estradiol at $26^{\circ} \mathrm{C}$ under a 16 -h light $\left(200 \mu \mathrm{mol} \mathrm{m} \mathrm{m}^{-2} \mathrm{~s}^{-1}\right)$ period; the medium was changed every 3 days (Fig. 7). 17- $\beta$-Estradiol (Sigma-Aldrich, St. Louis, MO, USA) was prepared as 50,10 , and $5 \mathrm{mM}$ stock solutions in dimethyl sulfoxide and stored at $-30^{\circ} \mathrm{C}$. The stock solution was added to the culture medium (N6D for calli and MS for seedling) with (N6D) or without (MS) the addition of $0.2 \%$ gelrite after autoclaving. 3-day-old seedlings were transferred onto square plates (No. 2 square scale, Eiken Chemical Co., Ltd., Tokyo, Japan) with MS solid medium containing $0.3 \%$ gelrite with or without estradiol at $26^{\circ} \mathrm{C}$ under a 16-h light period (Fig. 9).

\section{Detection of GFP fluorescence}

GFP fluorescence was observed using a fluorescence microscope with a GFP2 filter (480-nm excitation filter/ 510-nm barrier filter; Leica MZ 16FA, Leica Microsystems, Wetzlar, Germany).
PCR and Southern blot analysis

Genomic DNA was extracted from leaves or calli using the Isoplant II kit (Nippon gene, Tokyo, Japan) according to the manufacturer's protocol. PCR analysis was performed using Ex Taq DNA polymerase (Takara Bio Inc.) with the primer sets 5'-GAGCCTGACCTATTGCATCTC-3'/5'-GT ACTTCTACACAGCCATCG-3' for $h p t, 5^{\prime}$-CTGGGTGCT CAGGTAGTGG-3'/5'-CAGAAGAACGGCATCAAGG-3' for GFP, and 5'-GAAGTGCAAGAACGTGGTGC-3'/ 5'-GGAATGCGATGAAGTAGAGC-3' for XVE. Southern blot analysis was performed according to a standard protocol. A specific DNA probe for hpt was prepared using the PCR DIG labeling mix (Roche Diagnostics, Basel, Switzerland) with the abovementioned $h p t$-specific primer set. Southern blot hybridization signals were detected using the DIG luminescent detection kit (Roche Diagnostics).

Semi-quantitative RT-PCR analysis

Total RNA was isolated from rice calli using the RNeasy plant mini kit (Qiagen, Hilden, Germany). First-strand cDNA was synthesized from 500 ng of total RNA using the PrimeScript RT reagent kit (Takara Bio Inc.), oligo-dT primer, and random 6 mers according to the manufacturer's instructions. First-strand cDNA was used as the template for PCR with Takara Ex Taq DNA polymerase. PCR was performed for 25 cycles at $95^{\circ} \mathrm{C}$ for $15 \mathrm{~s}, 62^{\circ} \mathrm{C}$ for $15 \mathrm{~s}$, and $72^{\circ} \mathrm{C}$ for $20 \mathrm{~s}$ using the primer sets $5^{\prime}$-CTCTGTATG CCAGTGGTCG-3'/5'-GAGGTAATCAGTGAGATCAC-3' for OsAct1 (accession number X16280), 5'-CTGGGTGCT CAGGTAGTGG-3'/5'-CAGAAGAACGGCATCAAGG-3' for $G F P$, and 5'-GAAGTGCAAGAACGTGGTGC-3'/ 5'-GGAATGCGATGAAGTAGAGC-3' for XVE.

\section{Western blot analysis of the GFP protein}

Total soluble protein was extracted from $100 \mathrm{mg}$ calli, leaves, or roots in extraction buffer $(0.1 \mathrm{M}$ sodium phosphate, $0.01 \%$ ). Protein samples were separated on $15 \%$ $e$-PAGEL (ATTO Corporation, Tokyo, Japan). The gel was stained with Quick CBB (Wako) or transferred onto a Sequi-Blot PVDF membrane (Bio-Rad Laboratories, Hercules, CA, USA). Western blot analysis was performed using the ECL Plus Western blotting detection kit according to the manufacturer's protocol (GE Healthcare, Buckinghamshire, UK). Living colors Av (JL-8) monoclonal antibody (dilution of 1:4,000; Clontech, Mountain View, CA, USA) was used as the primary antibody, and a peroxidase-labeled anti-mouse antibody (dilution of 1:25,000; no. NIF 825, GE Healthcare) was used as the secondary antibody. The recombinant GFP protein purified 
from transformed Escherichia coli (Clontech) was used as a standard for protein quantification. Western blot hybridization signals were detected and analyzed using a Lumivision PRO system (TAITEC, Saitama, Japan). GFP signals were quantitated using a Bio-Rad Molecular Imager FX and analyzed using the Quantity One software (BioRad Laboratories).

\section{Results}

Production of transgenic rice integrated with $p L e x: G F P$

We constructed the estrogen-inducible GFP expression vector pLex:GFP (Fig. 1a) for rice using $p E R 8$ (Zuo et al. 2000a). To efficiently obtain transgenic rice, we used the binary vector $p Z H 2 B$ in which hpt is located close to the left border and driven by a $35 \mathrm{~S}$ promoter as a backbone.

Nineteen $p L e x: G F P$-integrated transgenic lines were obtained, and insertion of pLex:GFP was confirmed by PCR. Southern blot analysis with the hpt probe showed that 6 (\#1, \#3, \#10, \#17, \#21, and \#24) of the 19 lines contained a single copy of the introduced gene (Fig. 1b). To obtain positive control lines, we introduced p35S:GFP into rice and selected single copy integration by Southern blot analysis. To confirm the reliability of the estradiol-inducible system in transgenic rice, calli derived from the roots of $\mathrm{T}_{0}$ pLex:GFP-integrated transgenic rice were treated with $5 \mu \mathrm{M}$ estradiol for 2 days. GFP signals were detected in all calli treated with estradiol, but not in those that were not treated with estradiol (data not shown). These six lines having a single copy of pLex:GFP were grown, and their $\mathrm{T}_{1}$ seeds were obtained for further analysis.

Dose-response pattern of estradiol-inducible GFP expression

Calli derived from $\mathrm{T}_{1}$ seeds of pLex:GFP-integrated transgenic rice (\#1, \#3, \#10, \#17, \#21, and \#24) were proliferated in N6D culture media containing $30 \mathrm{mg} \mathrm{L}^{-1}$ hygromycin for 4 weeks without estradiol. For preliminary test of GFP induction in calli, propagated calli were cultured in N6D solid media containing $1 \mu \mathrm{M}$ estradiol for 2 days. Although GFP signals could be detected in calli derived from 3 lines of $\mathrm{T}_{0}(\# 10, \# 17$, and \#24) treated with estradiol, no GFP signal was detected in calli from $\mathrm{T}_{1}$ seeds; the presence of pLex:GFP in these three lines was confirmed by PCR. Another three lines (\#1, \#3, and \#21) had detectable GFP signals in calli derived from $T_{1}$ seeds treated with estradiol. Thus, these three lines (\#1, \#3, and \#21) were used for further analysis. Calli (5 $\mathrm{mm}$ in diameter) derived from a single seed of lines \#1, \#3, and \#21 were transferred to media containing seven different estradiol concentrations
$(0,0.01,0.1,1.0,5.0,10$, and $25 \mu \mathrm{M})$, and GFP fluorescence was observed after 48 h (Fig. 2). Within 12 h, GFP signals were first detected in the lower part of calli in contact with the media (data not shown). Later, the signals were detected in the entire surface of calli within $48 \mathrm{~h}$ (Fig. 2). In lines \#3 and \#21 treated with 0.01 and $0.1 \mu \mathrm{M}$ estradiol, GFP signals were scarcely detectable. The signals were slightly detectable in line \#1 treated with $0.01 \mu \mathrm{M}$ estradiol; weak signals were detected in the entire surface of calli treated with $0.1 \mu \mathrm{M}$ estradiol. GFP signals gradually weakened from the bottom to the top of calli treated with $1 \mu \mathrm{M}$ estradiol, and homogenous GFP signals were observed in the entire surface of calli treated with $5 \mu \mathrm{M}$ estradiol in all three lines. The highest level of GFP expression in the $p$ Lex:GFP-integrated transgenic calli did not exceed that in the p35S:GFP transgenic calli under all conditions (Fig. 2). The amount of GFP accumulated was later confirmed by Western blotting analysis (Fig. 4). Faint yellow fluorescence signals observed in the images of calli not treated with estradiol (\#1, \#3, and \#21) were background signals resulting from cell autofluorescence, and similar signals were detected in wild-type calli (Fig. 2). The pLex: GFP-integrated transgenic line \#1 conferred higher GFP signal intensities than the pLex:GFP-integrated transgenic lines \#3 and \#21 under all conditions (Fig. 2), and the pLex:GFP-integrated transgenic line \#1 was further analyzed.

GFP induction levels were also analyzed by semiquantitative RT-PCR and Western blot analysis. Semiquantitative RT-PCR showed a dose-dependent increase in

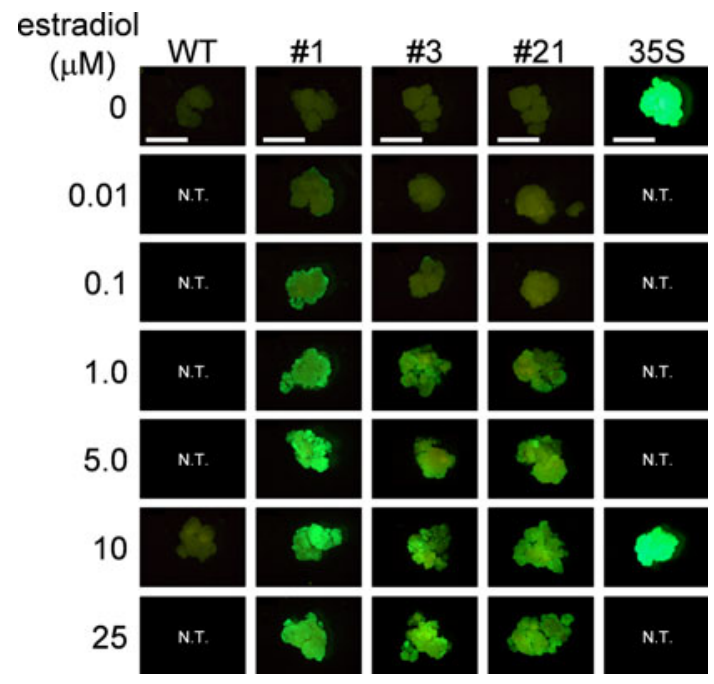

Fig. 2 GFP fluorescence image of the pLex:GFP-integrated transgenic calli $\left(\mathrm{T}_{1}\right)$ treated with estradiol. Calli derived from $\mathrm{T}_{1}$ seeds of the $p L e x: G F P$-integrated transgenic lines \#1, \#3, and \#21 were treated with $0,0.01,0.1,1.0,5.0,10$, and $25 \mu \mathrm{M}$ estradiol for $48 \mathrm{~h}$. Calli derived from wild-type (WT), non-transgenic calli, and p35S:GFPintegrated transgenic lines (35S) were also treated as negative and positive controls. N.T. not tested; Bar $5 \mathrm{~mm}$ 


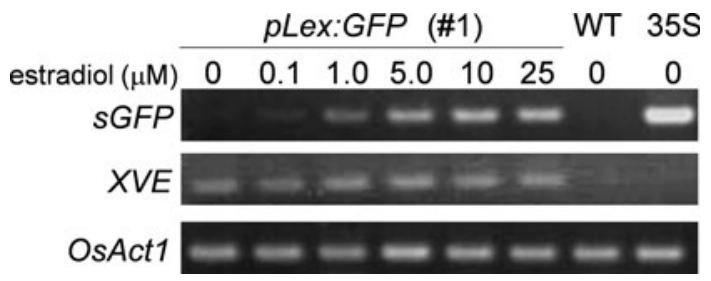

Fig. 3 Semi-quantitative RT-PCR analysis of the $s G F P$ and $X V E$ mRNA in the pLex:GFP-integrated transgenic calli. Total RNA was extracted from calli (\#1) treated with $0,0.1,1.0,5.0,10$, and $25 \mu \mathrm{M}$ estradiol. Calli derived from wild-type (WT) and p35S:GFPintegrated transgenic lines (35S) were analyzed as positive and negative controls

GFP transcripts of calli treated with $0.1,1.0$, and $5.0 \mu \mathrm{M}$ estradiol that appeared to be saturated at $10 \mu \mathrm{M}$ estradiol (Fig. 3). On the other hand, XVE transcript levels were stably detected under all conditions, including that for calli not treated with estradiol. In the same manner as observed for the GFP fluorescence level, GFP transcript levels in the pLex:GFP-integrated transgenic calli were lower than those in the $p 35 S: G F P$-integrated transgenic calli. Western blot analysis also showed a dose-dependent increase in GFP accumulation in the transgenic calli of the pLex:GFPintegrated line \#1 treated with 1.0, 5.0, and $25 \mu \mathrm{M}$ estradiol (Fig. $4 \mathrm{a}$ ). In the transgenic calli of the pLex:GFP-integrated line \#1 treated with $25 \mu \mathrm{M}$ estradiol, GFP accumulation was about $40 \%$ of that of the transgenic calli of the $p 35 S: G F P$-integrated line (Fig. $4 \mathrm{~b}$ ).

Time course of estradiol-inducible

GFP expression in calli

Calli derived from a single seed of the pLex:GFP-integrated transgenic line \#1 were cultured on N6D media with $1.0,5.0,10$, and $25 \mu \mathrm{M}$ estradiol for 10 days, and GFP fluorescence was observed (Fig. 5a). GFP fluorescence was not observed in calli not treated with estradiol during the 10-day culture period. With estradiol treatment at $1 \mu \mathrm{M}$, GFP signals were detected in the entire surface of calli within 3 days; GFP signals then gradually diminished daily. The signals were barely detected on day 10 . With estradiol treatment at 5, 10, and $25 \mu \mathrm{M}$, the highest GFP signals for each concentration was detected from day 2 in the entire surface of calli and continued for 2, 3, and 8 days, respectively. To determine whether the decline in signal could be due to estradiol degradation, a portion of the propagated calli was transferred to fresh media after 10 days of estradiol treatment, and GFP fluorescence was observed the following day (Fig. 5b). GFP signals were recovered within 1 day after transfer to fresh medium containing the same estradiol concentration. Then, to investigate whether estradiol treatment interruption could directly lead to a decline in induced GFP signals, the
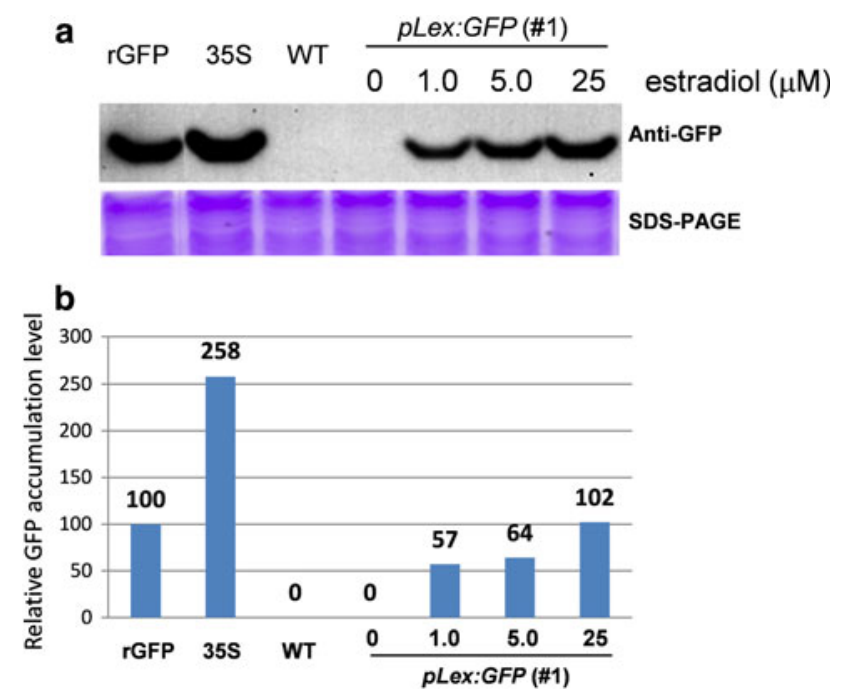

Fig. 4 Western blot analysis of calli harboring $p L e x: G F P$. a Total protein $(10 \mu \mathrm{g})$ extracted from the transgenic calli of the pLex:GFPintegrated line \#1 $\left(\mathrm{T}_{1}\right)$, which were treated with $0,1.0,5.0$, and $25 \mu \mathrm{M}$ estradiol for $48 \mathrm{~h}$, were separated by SDS-PAGE and analyzed using an anti-GFP antibody. $r G F P 50 \mathrm{ng}$ of the recombinant GFP protein purified from transformed Escherichia coli loaded with $10 \mu \mathrm{g}$ total protein extracted from calli of wild-type lines. WT total protein $(10 \mu \mathrm{g})$ extracted from wild-type lines, $35 \mathrm{~S}$ total protein $(10 \mu \mathrm{g})$ extracted from the p35S:GFP-integrated transgenic lines (35S). b The relative level of GFP accumulation. GFP signal was normalized for each fraction using the signal generated from $50 \mathrm{ng}$ of rGFP as standard

transgenic calli of the pLex:GFP-integrated lines \#1, \#3, and \#21 showing the highest level of GFP expression were transferred onto fresh N6D solid media without estradiol (Fig. 6). GFP signals were continuously detected on 1 day after estradiol treatment interruption, and the signals then decreased gradually. These signals could not be detected on day 7 after estradiol treatment interruption in 2 of the lines (\#3 and \#21) and on day 9 in line \#1.

\section{GFP induction in seedlings}

Three-leaf-old seedlings of the pLex:GFP-integrated transgenic $\left(\mathrm{T}_{1}\right)$ lines \#1 and \#21 were used to monitor GFP expression in seedlings by treatment with five concentrations of estradiol $(0,5,10,25$, and $50 \mu \mathrm{M})$. Wild-type and p35S:GFP-integrated transgenic lines were also used as negative and positive controls, respectively (Fig. 7a, d). The results of the $p$ Lex:GFP-integrated transgenic line \#21 are shown in Fig. 7. After 1 day, GFP signals were detected in the root tips of seedlings treated with $5 \mu \mathrm{M}$ estradiol (Fig. 7c) and all other tested concentrations. GFP expression was not detected in both $p L e x: G F P$-integrated transgenic lines \#1 and \#21 not treated with estradiol (Fig. 7b, e, h). After 5 days of estradiol treatment, GFP signals were detected in whole roots, particularly in newly 


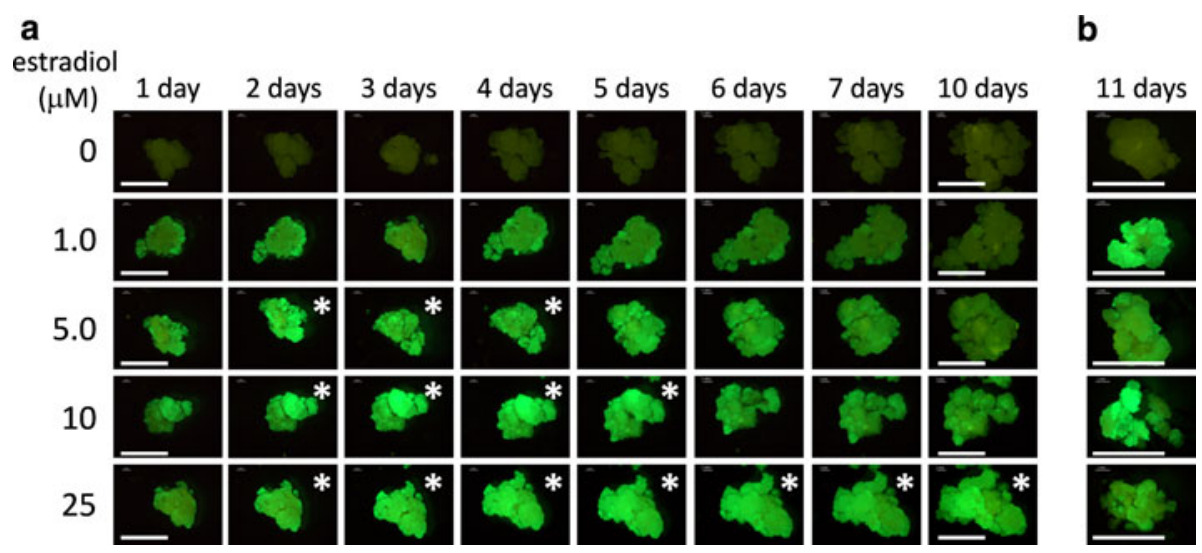

Fig. 5 Time course of GFP induction in pLex:GFP-integrated transgenic calli treated with estradiol. a Transgenic calli of the pLex:GFP-integrated line \#1 $\left(\mathrm{T}_{1}\right)$ were cultured on a solid medium with estradiol $(0,1.0,5.0,10$, and $25 \mu \mathrm{M})$, and GFP fluorescence was

elongated roots, at all concentrations (Fig. 7f, g). The signals were detected in a similar manner after 10 days (Fig. 7i, j). GFP signals could be barely detected in the leaves of the seedlings because of the presence of strong fluorescence signals from chlorophyll (such as the red color of the wild-type roots and bottom of the stem shown in Fig. 7a), which may have masked weak GFP signals (data not shown). We detected GFP accumulation by Western blot analysis in both the roots and leaves after 5 and 10 days of estradiol treatment (Fig. 8). GFP accumulation in the leaves of the pLex:GFP-integrated transgenic seedlings treated with estradiol were much lower than those of the $p 35 S: G F P$-integrated transgenic seedlings, and the signals were slightly weaker on day 10 (Fig. 8). Faint GFP signals were also detected on day 10 in the leaves of the seedlings not treated with estradiol. In the roots of the pLex:GFP-integrated transgenic seedlings treated with estradiol, GFP accumulated to the same level as in the p35S:GFP-integrated transgenic seedlings, except with

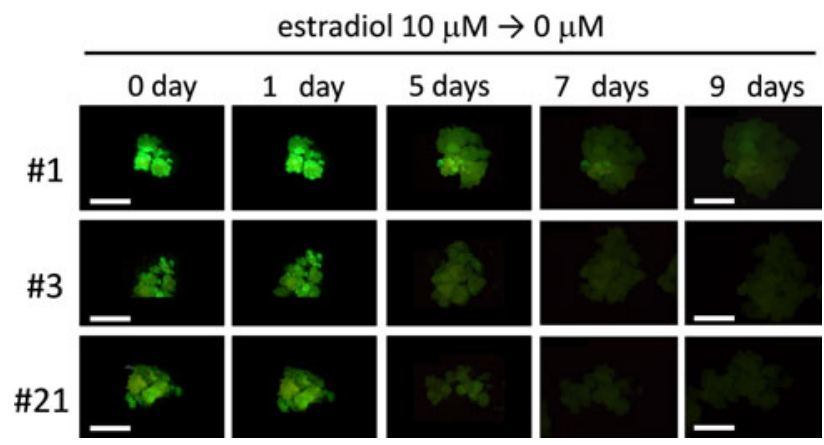

Fig. 6 Estradiol treatment interruption after GFP induction for $48 \mathrm{~h}$. Transgenic calli derived from $\mathrm{T}_{1}$ seeds of the pLex:GFP-integrated lines (\#1, \#3, and \#21) were treated with $10 \mu \mathrm{M}$ estradiol in culture media for $48 \mathrm{~h}$. They were then transferred to MS medium without estradiol (0 day) and cultured for 9 days. Bar $5 \mathrm{~mm}$ detected for 10 days. Calli estimated to have the highest level of GFP expression are marked with an asterisk (*). Bar $5 \mathrm{~mm}$. b A portion of the calli was transferred to a fresh medium and cultured for 1 day. Bar $5 \mathrm{~mm}$

estradiol treatment at $5 \mu \mathrm{M}$, and the same level of GFP accumulation was also detected on day 10 for all estradiol concentrations. Moreover, GFP signals were not detected in the roots of the seedlings not treated with estradiol (Fig. 8).

Phenotypic and growth analysis

of $p L e x: G F P$-integrated transgenic rice

The XVE system had no toxic or non-specific physiological effects on the host Arabidopsis (Zuo et al. 2000a), unlike the glucocorticoid-inducible GVG system, which can cause
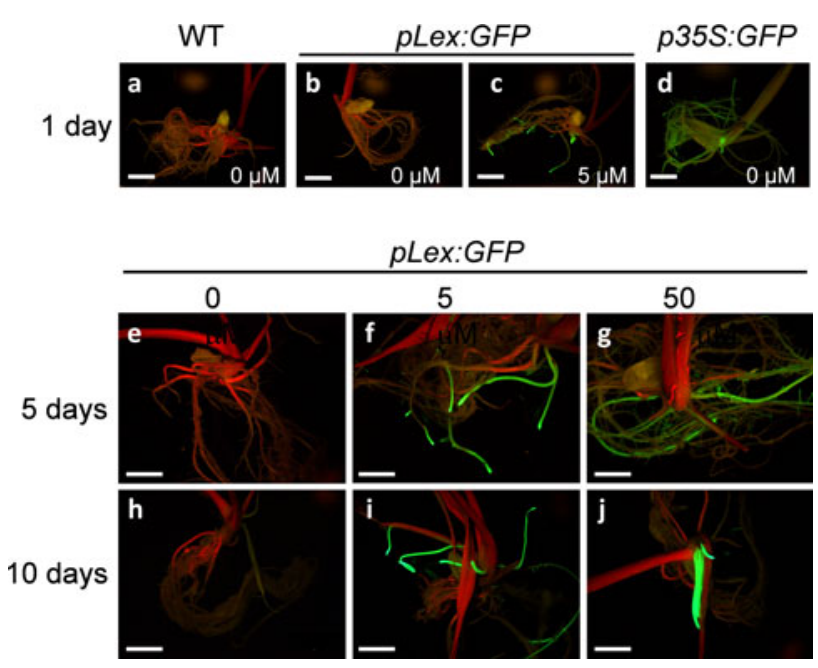

Fig. 7 GFP fluorescence image of the roots of seedlings treated with estradiol. a Wild-type (WT) lines not treated with estradiol. b, c The pLex:GFP-integrated transgenic line \#21 treated with 0 or $5 \mu \mathrm{M}$ estradiol for a day. d $p 35 S: G F P$-integrated transgenic lines (35S) not treated with estradiol. e-g The pLex:GFP-integrated transgenic line \#21 treated with 0,5 , or $50 \mu \mathrm{M}$ estradiol for 5 days. $\mathbf{h}-\mathbf{j}$ The pLex:GFP-integrated transgenic line \#21 with 0,5 , or $50 \mu \mathrm{M}$ estradiol for 10 days. Bar $5 \mathrm{~mm}$ 


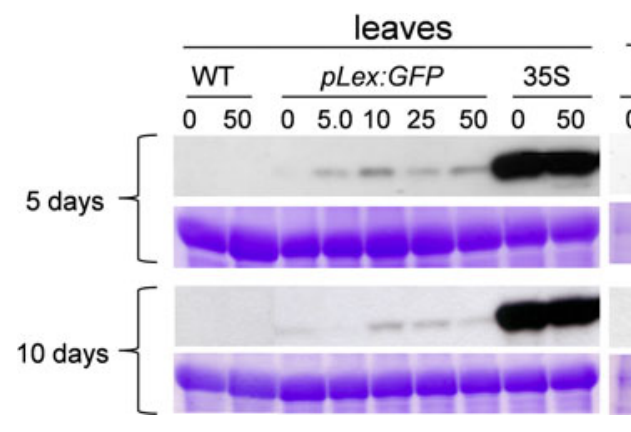

Fig. 8 Western blotting analysis of pLex:GFP-integrated transgenic seedlings. Three-leaf-stage transgenic seedlings of the pLex:GFPintegrated line \#21 (T1) were treated with $0,5,10,25$, and $50 \mu \mathrm{M}$ estradiol. Wild-type (WT) and p35S:GFP-integrated transgenic lines (35S) were treated with 0 and $50 \mu \mathrm{M}$ estradiol, respectively. After 5

growth defects in Arabidopsis (Kang et al. 1999) and rice (Ouwerkerk et al. 2001). To observe phenotypic effects of the XVE system, 4-day-old seedlings were transferred to MS solid media containing $10 \mu \mathrm{M}$ estradiol and cultured for 10 days. Roots of all lines ( $p$ Lex:GFP, wild type, and p35S:GFP) treated with estradiol had more root hairs and their root tips were less winding compared to those of lines not treated with estradiol. However, other morphological differences were not observed in the $p L e x: G F P$-integrated transgenic and control lines after estradiol treatment (Fig. 9). Furthermore, plant height and root length of the pLex:GFP-integrated transgenic lines were not significantly different for estradiol treatment at 0 and $10 \mu \mathrm{M}$ (plant height, $13.0 \pm 2.0$ vs. $14.0 \pm 2.3$; root length, $3.3 \pm 0.8$ vs. $4.3 \pm 0.8 ; n=3$ ) according to the $t$ test $(P>0.05)$ performed after 10 days of estradiol treatment.

\section{Discussion}

Effective target gene induction in rice

In a previous study using the XVE system in rice, Sreekala et al. (2005) showed that marker-free transgenic plants are produced by the induction of Cre recombinase with estradiol treatment at $10 \mu \mathrm{M}$ for 2 weeks in calli; however, they did not show the expression levels of Cre recombinase. In another study, Xu et al. (2009) showed that the target gene is suppressed using an induced RNA interference system in plants treated with $5 \mu \mathrm{M}$ estradiol for 12 days, and the level of target gene suppression were shown. Since little information is available on the levels of target gene expression by the XVE system in rice, we believe that a thorough knowledge of the correlation between the efficacy of estradiol treatment and gene expression levels is important for various studies. To clarify the gene induction patterns, we produced $p L e x: G F P$-integrated transgenic rice

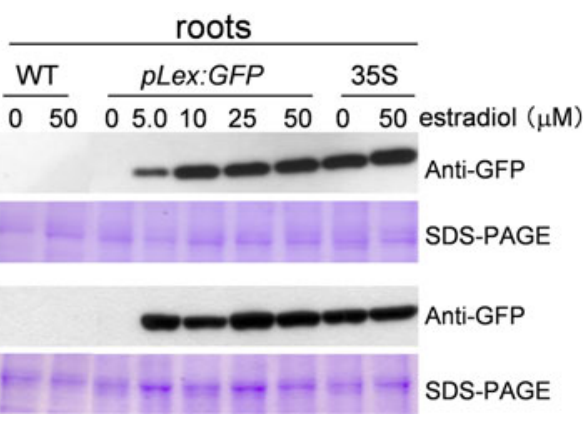

and 10 days of estradiol treatment, total soluble proteins were extracted from the leaves and roots of the seedlings. Total soluble protein from the leaves $(20 \mu \mathrm{g})$ and roots $(5 \mu \mathrm{g})$ were separated by SDS-PAGE and analyzed using an anti-GFP antibody

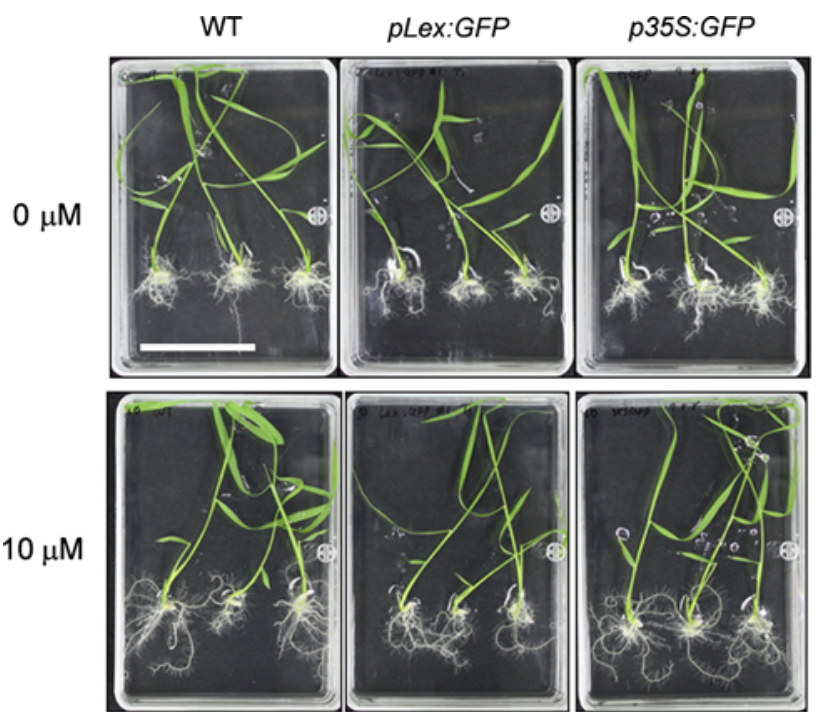

Fig. 9 Phenotypes of pLex:GFP-integrated transgenic seedlings treated with and without estradiol. Seeds of wild-type (WT; left) lines, the pLex:GFP-integrated transgenic line \#1 (middle), and p35S:GFP-integrated transgenic lines (right) were germinated on MS media without estradiol for 3 days, and then cultured on MS media containing 0 or $10 \mu \mathrm{M}$ estradiol for 7 days. Bar $5 \mathrm{~cm}$

plants. In the XVE system in Arabidopsis, saturated GFP transcript levels were four times higher than those in p35S:GFP-integrated transgenic plants (Zuo et al. 2000a). In our study using $p L e x: G F P$-integrated transgenic rice, we detected target gene induction by visual observation and molecular analysis. The highest GFP accumulation in the roots of the $p L e x: G F P$-integrated transgenic seedlings was similar to that in the roots of the p35S:GFP-integrated transgenic seedlings (Fig. 8). However, in calli and leaves, the highest GFP accumulation observed in the pLex:GFPintegrated transgenic lines was lower than that in the p35S:GFP-integrated transgenic lines (Figs. 4, 8). The two possible factors responsible for low levels of target gene induction in rice leaves are an inefficient estradiol uptake 
and a weak G10-90 promoter activity in these leaves resulting in low XVE expression levels. Estradiol uptake seems to be a crucial factor for a functional XVE system. In our study, the XVE system rapidly induced the target gene and a stable expression of this gene was obtained in rice calli and roots, while weaker GFP signals were detected in leaves even with estradiol treatment at $50 \mu \mathrm{M}$, which is a concentration 10 times higher than that required for the highest level of GFP expression in roots (Fig. 8). Cut leaf segments of the pLex:GFP-integrated transgenic lines, which were cut on both sides to obtain a size of $2-3 \mathrm{~cm}$ and placed in MS liquid media with $10 \mu \mathrm{M}$ estradiol, rapidly showed visually detectable GFP fluorescence in whole leaf segments within $48 \mathrm{~h}$ (data not shown). This result indicates that the XVE system can respond to induction by estradiol treatment even in the leaves of the pLex:GFP-integrated transgenic lines; however, there seems to be any limiting factor of target gene expression in leaves even when the lines were treated with sufficient estradiol concentration from roots. Although the calli and roots were in direct contact with the culture medium and could absorb enough estradiol under the test conditions, estradiol uptake in the leaves was dependent on the roots. Since plant height increased and leaf area expanded about 3 times during estradiol treatment, absorbed estradiol might have been diffused and attenuated. Furthermore, estradiol may have been trapped by XVE expressed in roots, and the estradiol concentration in leaves was probably reduced and did not reach adequate high concentrations. Similar results of reduced induction in leaves have been shown in the GVG system in which flowers required ten times more dexamethasone $(100 \mu \mathrm{M})$ to induce sufficient target gene expression compared with 6-day-old young seedlings (Ouwerkerk et al. 2001). Another possible reason could be that XVE expression levels resulting from the G10-90 synthetic promoter activity affected induction levels. The G10-90 promoter, which is a modified promoter of the CaMV 35S promoter, confers a high-level constitutive expression in transgenic tobacco (Ishige et al. 1999). Thus, highly expressed XVE by constitutive G10-90 synthetic promoter could effectively respond to estradiol in dicotyledonous Arabidopsis (Zuo et al. 2000a). However, the G10-90 promoter activity in rice seems to be lower than in tobacco (Ishige et al. 1999). Therefore, estradiol concentration and XVE expression levels seem to act synergistically and lead to insufficient GFP expression levels in rice leaves. Xu et al. (2009) reported that only 2 of 40 lines successfully suppress the target gene, and these 2 lines had integrated multiple copies of the vector. Multicopy integration may favor XVE expression in response to estradiol. The GVG system has been demonstrated to quickly respond to the inducer in rice, because the rice Gos 2 promoter confers high levels of constitutive GVG expression in all parts of the plant (Ouwerkerk et al. 2001). If $X V E$ was linked to the Gos 2 promoter or other strong constitutive expression promoters, such as the ubiquitin promoter (Wang and Oard 2003) or the recently examined promoters $A P X, P G D 1$, and RlGlB from rice (Park et al. 2010), the response to estradiol would increase in rice or other monocotyledonous plants. The choice of a high, weak, or site-specific expressing promoter of $X V E$ will depend on the characteristics of the target gene and the needs of the study conducted. The G10-90 promoter should be useful for mild induction of a target gene such as lethal genes in rice.

Stable induction and interruption of induction in calli

We next observed continuous gene induction during cell cultures. The period of expression of the highest GFP signal was extended in calli cultured on a solid medium at higher estradiol concentrations (Fig. 5a). Decrease in GFP signals with $10 \mu \mathrm{M}$ estradiol could be recovered when calli were transferred to a fresh medium (Fig. 5b). Thus, decreased GFP expression in the calli appeared to result from estradiol depletion or degradation in the media; the XVE system was not suppressed. These results possibly indicate that increased estradiol concentration or frequent change of the solid medium is effective in stably and continuously inducing the target gene in calli cultured on solid media. In addition, interruption of target gene induction in calli was demonstrated. Calli expressing the highest GFP signals were transferred to fresh media without estradiol, and GFP signals in such calli gradually decreased and disappeared after 7-9 days (Fig. 6). Several days after estradiol treatment was suspended, estradiol was probably still present in the calli, resulting in weak GFP expression for several days, since the reported half-life of GFP is $18 \mathrm{~h}$ (de Ruijter et al. 2003).

\section{The XVE system using the $p L e x: G F P$ construct}

We selected six transgenic rice lines with a single copy of pLex:GFP for further analysis (Fig. 1b). Zuo et al. (2000a) reported that some transgenic lines containing the inducible $G F P$ vector do not show any detectable GFP signals or show a patchy-type expression, presumably caused by a positional effect on the transgene. In our study, six transgenic rice lines with a single copy of $p$ Lex:GFP showed detectable GFP signals in the calli of $\mathrm{T}_{0}$, however, calli derived from $T_{1}$ seeds of the three lines (\#10, \#17 and \#24) did not show any detectable GFP signal. This could be due to gene silencing. The other three lines (\#1, \#3, and \#21) showed detectable GFP signals upon estradiol treatment, which did not lead to significant morphological differences compared to that in wild-type lines (Figs. 5, 9). Plant height and root 
length of the seedlings were not significantly different as confirmed by the $t$ test. Although the XVE system had no effect on growth, all tested lines (wild type, $p L e x: G F P$, and p35S:GFP) presented a different root spread pattern with estradiol treatment (Fig. 9). We concluded that this could be due to the addition of estradiol to the media. Consequently, the XVE system, which uses estradiol as an inducer, does not appear suitable for root morphology studies. GFP signals were not detected in the pLex:GFP-integrated transgenic calli and seedlings (roots) (Figs. 2, 3, 4), whereas faint GFP expression was detected by Western blot analysis in the leaves of the seedlings not treated with estradiol (Fig. 8). Considering the faint expression in rice leaf, single copyintegrated plants may be required to reduce the leaky expression effect, particularly for the induction of lethal gene expression using this vector system.

\section{Availability of the XVE system in rice}

In conclusion, we investigated target gene expression patterns in rice using the XVE system and demonstrated the availability of this system in rice calli and seedlings for the first time. Target gene induction levels were dependent on not only estradiol concentration but also constitutive expression levels of $X V E$ in transgenic rice. Moreover, $G F P$ induction levels and patterns in seedlings were different between Arabidopsis and rice. These results might provide helpful information that could contribute to a wider use of the XVE system in order to control the target gene expression level in rice and other monocots.

Acknowledgments The authors are grateful to Dr. N.-H. Chua for providing $p E R 8$ and Dr. M. Kuroda for providing $p Z H 2 B$ and $p U C 198 A M$. We thank Drs. M. Mori, S. Toki, H. Saika, and S. Takahashi for technical support and advice. We also thank Ms. K. Masuda, K. Sasaki, C. Ito, I. Kawaguchi, and H. Fujita for technical support with regard to plant cultures. This study was supported by a grant from the Ministry of Agriculture, Forestry and Fisheries of Japan (Assurance of Safe Use of Genetically Modified Organisms Project).

Open Access This article is distributed under the terms of the Creative Commons Attribution Noncommercial License which permits any noncommercial use, distribution, and reproduction in any medium, provided the original author(s) and source are credited.

\section{References}

Aoyama T, Chua NH (1997) A glucocorticoid-mediated transcriptional induction system in transgenic plants. Plant J 11(3): 605-612

Chu CC, Wang CC, Sun CS, Hsu C, Yin KC, Chu CY, Bi FY (1975) Establishment of an efficient medium for anther culture of rice through comparative experiments on the nitrogen sources. Sci Sinica 18:25-31
Costa LD, Vaccari I, Mandolini M, Martinelli A (2009) Elaboration of a reliable strategy based on Real-Time PCR to characterize genetically modified plantlets and to evaluate the efficiency of a marker gene removal in grape (Vitis spp.). J Agric Food Chem 57:2668-2677

de Ruijter NCA, Verhees J, van Leeuwen W, van der Krol AR (2003) Evaluation and comparison of the GUS, LUC and GFP reporter system for gene expression studies in plants. Plant Biol 5:103-115

Dohi K, Nishikiori M, Tamai A, Ishikawa M, Meshi T, Mori M (2006) Inducible virus-mediated expression of a foreign protein in suspension-cultured plant cells. Arch Virol 151:1075-1084

Hajdukiewicz P, Svab Z, Maliga P (1994) The small, versatile $p P Z P$ family of Agrobacterium binary vectors for plant transformation. Plant Mol Biol 25:989-994

Hood EE, Gelvin SB, Melchers LS, Hoekema A (1993) New Agrobacterium helper plasmids for gene transfer to plants. Transgenic Res 2:208-218

Ishige F, Takaichi M, Foster R, Chua NH, Oeda K (1999) A G-box motif (GCCACGTGCC) tetramer confers high-level constitutive expression in dicot and monocot plants. Plant J 18(4):443-448

Kang HG, Fang Y, Karam BS (1999) A glucocorticoid-inducible transcription system causes severe growth defects in Arabidopsis and induces defense-related genes. Plant J 20:127-133

Klimaszewska K, Pelletier G, Overton C, Stewart D, Rutledge RG (2010) Hormonally regulated overexpression of Arabidopsis WUS and conifer LEC1 (CHAP3A) in transgenic white spruce: implications for somatic embryo development and somatic seedling growth. Plant Cell Rep 29:723-734

Kuroda M, Kimizu M, Mikami C (2010) A simple set of plasmids for the production of transgenic plants. Biosci Biotechnol Biochem 74(11):2348-2351

Niwa Y, Hirano T, Yoshimoto K, Shimizu M, Kobayashi H (1999) Non-invasive quantitative detection and applications of nontoxic, S65T-type green fluorescent protein in living plants. Plant J 18:455-463

Ouwerkerk PBF, de Kam RJ, Hoge HC, Meijer AH (2001) Glucocolticoid-inducible gene expression in rice. Planta 213:370-378

Padidam M (2003) Chemically regulated gene expression in plants. Curr Opin Plant Biol 6:169-177

Park SH, Yi N, Kim YS, Jeong MH, Bang SW, Choi YD, Kim JK (2010) Analysis of five novel putative constitutive gene promoters in transgenic rice plants. J Exp Bot 61(9):2459-2467

Petrasek J, Mravec J, Bouchard R, Blakeslee JJ, Abas M, Seifertova D, Wisniewska J, Tadele Z, Kubes M, Covanova M, Dhonukshe P, Skupa P, Benkova E, Perry L, Krecek P, Lee RO, Fink RG, Geisler M, Murphy SA, Luschnig C, Zazimalova E, Friml J (2006) PIN proteins perform a rate-limiting function in cellular auxin efflux. Sience 312:914-918

Sreekala C, Wu L, Gu K, Wang D, Tian D, Yin Z (2005) Excision of a selectable marker in transgenic rice (Oryza sativa L.) using a chemically regulated Cre/loxP system. Plant Cell Rep 24:86-94

Tang W, Luob X, Samuels V (2004) Regulated gene expression with promoters responding to inducers. Plant Sci 166:827-834

Toki S, Hara N, Ono K, Onodera H, Tagiri A, Oka S, Tanaka H (2006) Early infection of scutellum tissue with Afrobacterium allows high-speed transformation of rice. Plant J 47:969-979

Wang J, Oard JH (2003) Rice ubiquichin promoter: deletion analysis and potential usefulness in plant transformation systems. Plant Cell Rep 22:129-134

$\mathrm{Xu}$ M, Dong JF (2007) Enhancing terpenoid indole alkaloid production by inducible expression of mammalian Bax in Catharanthus roseus cells. Sci China Ser C-Life Sci 50:234-241

Xu H, Zhang J, Zeng J, Jiang L, Liu E, Peng C, He Z, Peng X (2009) Inducible antisense suppression of glycolate oxidase reveals its 
strong regulation over photosynthesis in rice. $\mathrm{J}$ Exp Bot 60(6):1799-1809

Zhang Y, Li H, Ouyang B, Lu Y, Ye Z (2006) Chemical-induced autoexpression of selectable markers in elite tomato plants transformed with a gene conferring resistance to lepidopteran insects. Biotechnol Lett 28:1247-1253

Zuo J, Chua NH (2000) Chemical-inducible systems for regulated expression of plant genes. Curr Opin Biotechnol 11(2):146-151

Zuo J, Niu QW, Chua NH (2000a) An estrogen receptor-based tranactivator XVE mediates highly inducible gene expression in transgenic plants. Plant J 24:265-273
Zuo J, Niu QW, Nishizawa N, Wu Y, Kost B, Chua NH (2000b) KORRIGAN, an Arabidopsis endo-1, 4- $\beta$-glucanase, localizes to the cell plate by polarized targeting and is essential for cytokinesis. Plant Cell 12:1137-1152

Zuo J, Niu QW, Moller SG, Chua NH (2001) Chemical-regulated, site-specific DNA excision in transgenic plants. Nat Biotechnol 19:157-161 